\title{
Primary actinomycotic lung abscess
}

\author{
A Mohan, TK Chattopadhyay, SK Sharma, AK Dinda, R Sharma
}

\begin{abstract}
Summary
A case of recurrent massive haemoptysis in whom primary actinomycotic lung abscess was diagnosed following right pneumonectomy is reported.
\end{abstract}

Keywords: lung abscess, primary actinomycosis

Actinomycosis is a chronic infectious disease characterised by suppuration, purulent discharge containing sulphur granules, and sinus tract formation. The disease usually presents in the cervicofacial, abdominopelvic and thoracic forms. We report a case of primary actinomycotic lung abscess.

\section{Case report}

A 20-year-old man presented with a history of low-grade intermittent fever, cough with copious foul-smelling purulent sputum, recurrent massive haemoptysis and right-sided pleuritic chest pain for the preceeding two years. Before coming to hospital, he was diagnosed to have pulmonary tuberculosis on the basis of his clinical features and chest X-ray and had been receiving antituberculosis treatment for the last 13 months with rifampicin (R), isoniazid $(\mathrm{H})$, ethambutol (E) and streptomycin (S) in the combination HRE for the initial two months, SHRE for the next three months and RH since then. His sputum, however, did not reveal Mycobacterium tuberculosis on smear and culture examination on six occasions. He had also undergone bronchial biopsy which was inconclusive and revealed nonspecific inflammatory changes. He did not have any associated illness.

He presented to the emergency room with massive haemoptysis and was admitted. Following admission, physical examination revealed that he was of moderate build and poorly nourished. He had severe pallor and digital clubbing. His pulse rate was 110 beats/ min, respirations $32 / \mathrm{min}$ and blood pressure was $105 / 80 \mathrm{mmHg}$. Respiratory system examination revealed right upper lobe consolidation. The rest of the physical examination was unremarkable.

Investigations revealed: haemoglobin $2.7 \mathrm{~g}$ $\mathrm{dl}$; total leukocyte count $12 \times 10^{9} / 1$, differential count of polymorphs $55 \%$ and lymphyocytes $40 \%$; erythrocyte sedimentation rate was $55 \mathrm{~mm} / \mathrm{h}$ (Westergren method). Routine blood chemistry was normal. Sputum smear did not reveal acid-fast organisms. Sputum culture did not grow Mycobacterium tuberculosis, pathogenic fungi or pyogenic bacteria. Serum precipitins against Aspergillus fumigatus were negative. A chest X-ray (figure 1) and contrastenhanced computed tomography (CT) chest scan (figure 2) were performed. Pulmonary functions revealed a vital capacity of 2.861 (predicted value $=4.30 \mathrm{l}$ ); ratio of forced expiratory volume in 1 second to forced vital capacity $=96 \% \quad$ (predicted value $=80 \%$ ); maximum mid-expiratory flow rate $=3.41 \mathrm{l} / \mathrm{s}$ (predicted value $=4.41 \mathrm{l} / \mathrm{s}$ ).

In view of the persistent recurrent uncontrolled massive haemoptysis, he was transfused with packed red blood cells to build up his haemoglobin to $9 \mathrm{~g} / \mathrm{dl}$ and was taken up for surgery. The right chest was entered after clearing significantly dense fibrotic adhesions. The right middle lobe was inseparable from the lower lobe, was adherent to the upper lobe and felt firm and solid. Initial attempts at separating the upper lobe were abandoned due to increasing bleeding and soiling of the pleural cavity. Subsequently, the entire right lung was mobilised and removed after securing the right main pulmonary artery, superior and inferior pulmonary veins and the right main bronchus in that order. The specimen when sectioned revealed extremely thickened pulmonary

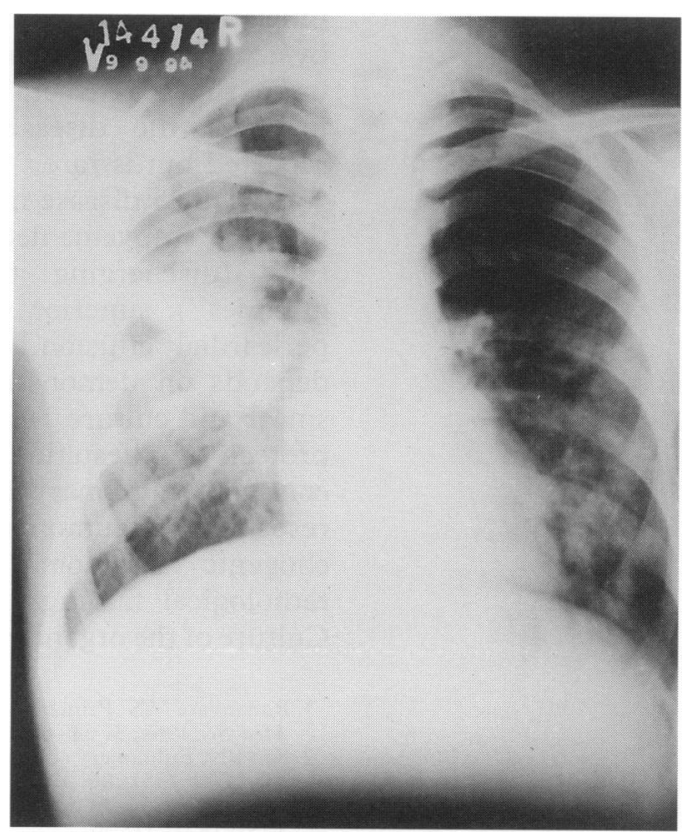

Figure 1 Chest X-ray posteroanterior veiw showing consolidation in right upper and middle lobe. Patchy consolidation was also seen in the lingula and the left lower lobe 


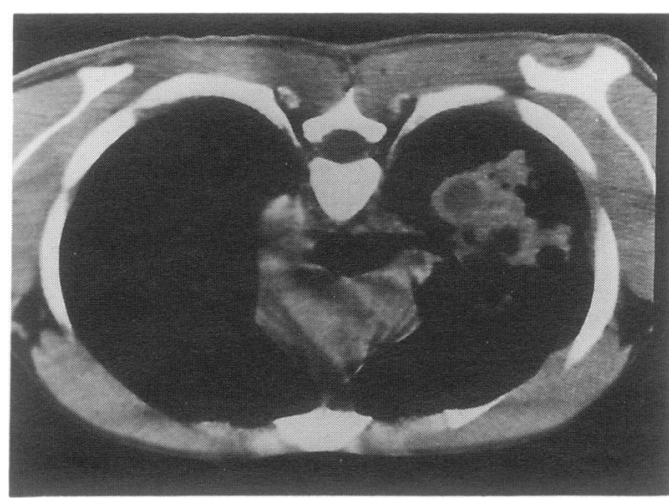

Figure 2 Contrast enhanced chest CT showing right upper lobe consolidation with breakdown and a fluid density lesion with rim enhancement

parenchyma overlying a deep-seated abscess cavity containing thick inspissated purulent material mixed with old blood clots. The abscess cavity, on histopathological examination (figure 3) showed a colony of Grampositive non-acid fast filamentous organisms compatible with actinomycetes. Lymph node dissected from the specimen showed reactive changes and no granulomas were seen in any of the sections examined. The patient received parenteral ampicillin, gentamicin, and metronidazole during the in-hospital stay. The chest X-ray at the time of discharge showed near complete resolution of the parenchymal lesions in the left lower zone. Presently, the patient is not on any treatment and is doing well.

\section{Discussion}

Primary actinomycotic lung abscess is an uncommon form of thoracic actinomycosis and is usually caused by Actinomycetes israeli. The organism is believed to enter the lung either by inhalation of contaminated aerosol particles or by aspiration of contaminated matter from the upper digestive tract. The primary pulmonary form of the disease has been previously reported but is rare. ${ }^{1-4}$

Advanced disease may present as empyema thoracis, empyema neccesitatis, draining sinus tract (discharging the classical "sulphur granules"), superior vena cava syndrome or pericardial effusion. ${ }^{5}$ A definitive diagnosis depends on demonstrating the organism on smear and culture. The disease, however, has protean manifestations and can mimic many common pulmonary diseases including nonresolving pneumonia, tuberculosis, bronchogenic carcinoma and infarction. The radiological findings are not specific either. Culture of the organism is either not performed

1 Rananvare MN. Primary actinomycosis of the lung. Indian $f$ Med Sci 1951; 5: 63-6.

2 Sen SN. Pulmonary actinomycosis. Report of a case. Indian Med Gaz 1951; 86: 101-3.

3 Subrmanian R. Actinomycosis of the lung. 7 Indian Med Assoc 1952; 21: 367.

4 Koshi G, Lalitha MK, Samraj T, Mathai KV. Brain abscess and other protean manifestations of actinomycosis. $A m \mathcal{F}$ Trop Med Hyg 1981; 30: 139-44.

5 Scully RE, Mark EJ, McNeely BU. Case records of the Massachusetts General Hospital: case 45. $N$ Engl $\mathcal{F}$ Med Massachusetts Gen

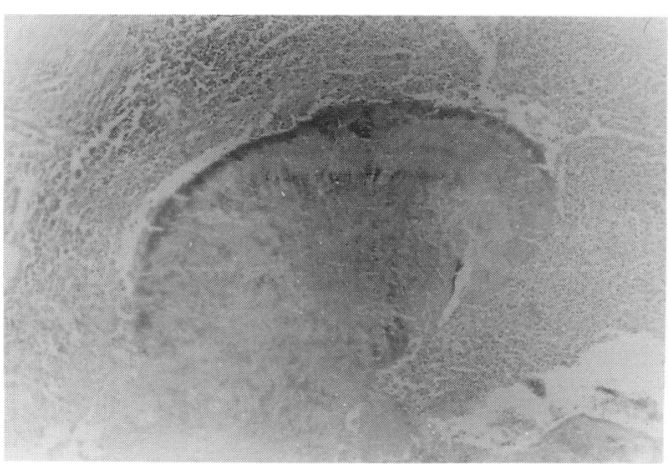

Figure 3 Photomicrograph showing a part of the eosinophilic granular substance with haematoxyphil bodies ('sulphur granule') surrounded by acute inflammatory exudate. (Haematoxylin and eosin $\times 100$ )

\section{Learning points}

- primary actinomycotic lung abscess is an uncommon form of thoracic actinomycosis and is usually caused by Actinomycetes israeli

- accurate diagnosis of this treatable condition is rarely made at admission owing to the nonspecific clinical and radiological findings.

- culture of the organism is either not performed at all or is incorrectly carried out without considering the microaerophilic requirements of the organism.

- when a patient suspected to have pulmonary tuberculosis remains sputum negative and does not respond to antituberculosis treatment (especially in areas where tuberculosis is prevalent), rare infections including actinomycosis must be considered.

- primary actinomycotic lung abscess can be a rare cause of life-threatening haemoptysis.

at all or is incorrectly carried out without considering the micro-aerophilic requirements of the organism. Therefore, accurate diagnosis of primary pulmonary actinomycosis is rarely made at the time of admission and is frequently made on histopathology rather than by microbiological methods. ${ }^{6}$ The present patient was diagnosed to have pulmonary tuberculosis but the diagnosis was not clear until the histopathology confirmed it.

Penicillin remains the drug of choice for treatment and may have to be given for several weeks depending on the extent and severity of the disease. Surgical intervention is usually performed to ascertain the diagnosis, obtain material for culture or histological examination or rule out other conditions which the disease simulates. ${ }^{78}$ Complications of the disease such as haemoptysis, empyema or chronic sinus discharge may, however, necessitate surgical intervention. ${ }^{7,9}$

6 Hsieh M-J, Liu H-P, Chang C-H. Thoracic actinomycosis Chest 1993; 104: 366-70.

7 Jara FM, Toledo-Pereyra LH, Magilligen DJ. Surgical implications of pulmonary actinomycosis. $\mathcal{f}$ Thorac Cardiovasc Surg 1982; 83: 218-26.

8 Halseth WL, Reith MP. Pulmonary actinomycosis treated by lung resection. Dis Chest 1969; 55: 119-22.

9 Smith DC, Lockwood WR. Disseminated actinomycosis. Chest 1975; 67: 242-4. 